\title{
Eye Movement Desensitization and Reprocessing desde la Propuesta de Valoración de Mario Bunge
}

\section{Eye Movement Desensitization and Reprocessing from Mario Bunge's assessment proposal}

\begin{abstract}
María de Lourdes Cedillo Armijos ${ }^{1}$, Juan Pablo Viñanzaca López ${ }^{2}$, María José Espinosa Valdivieso ${ }^{3}$, Rosa Estefanía Gómez Espinoza ${ }^{4}$, Christian Gustavo Mena Avila ${ }^{5}$, Estela Isabel Galarza Villavicencio ${ }^{6}$, Pedro Carlos Martínez-Suárez ${ }^{7}$, Andrés Alexis Ramirez-Coronel ${ }^{8}$

INFORMACIÓN DEL

ARTÍCULO

Fecha de recepción: agosto 20,2020.

Fecha de aceptación: enero 18, 2021.

Unidad de Diagnóstico, Investigación Psicopedagógica y Apoyo a la Inclusión, Universidad Católica de Cuenca. PhD(c) por la Pontificia Universidad Católica del Perú. E-mail:mcedilloa@ucacue.edu.ec Código ORCID:

https://orcid.org/0000-0002-6114-5305

2 Unidad Educativa Particular Católica do Cuenca, Universidad Católica de Cuence, Cuenca, Universidad Católica de Cuenca,
Maestría en Neuropsicología y Educación por Maestria en Neuropsicologia y Educacion por
la Universidad de la Rioja; maestrante en la Universidad de la Rioja; maestrante en
Psicología Clínica con mención en Psicoterapia por la Universidad Católica de Cuenca. E-mail: juanpablofishitos@ @gmail.com Código ORCID

https://orcid.org/0000-0003-0238-4522

3 Maestrante en Psicologí Clínica con Maestrante en Psicología Clínica con mención en Psicoterapia por la Universida Católica de Cuenca.

E-mail: mari.jose1903@hotmail.com

https:/0rcidorg/0000-0002-9503-1519

4 Maestrante en Psicología Clínica con mención en Psicoterapia por la Universidad

\section{Resumen}

La práctica de la intervención psicológica debería estar basada en las mejores evidencias científicas acerca de su eficacia. El objetivo de este estudio fue analizar la actitud, validez y dominio científico que posee la psicoterapia de la Desensibilización y Reprocesamiento por los Movimientos Oculares (EMDR) con base en un enfoque de realismo científico propuesto por Bunge en su tabla de comparación de las actitudes científicas y pseudocientíficas. El diseño metodológico incluyó la adaptación lingüística y cultural, la validación de contenido con el criterio de jueces, de la tabla de Bunge; la búsqueda bibliográfica de artículos entre 2016-2020, en las bases de datos de Scopus y Web of Science, con diseños de estudio experimentales y cuasiexperimentales relacionados al EMDR. Los hallazgos evidenciaron 2417 publicaciones, 38 de las cuales cumplen con los criterios señalados, en donde se indica que la EMDR reflejan los criterios para considerarse una ciencia, sosteniendo su eficacia.

Palabras Clave:

Ciencia, Pseudociencia, EMDR, Tabla Bunge, Validación.

Clasificación JEL: Z00, Z19.
\end{abstract} Católica de Cuenca.

E-mail: estefy2224@outlook.es

Código ORCID:

https://orcid.org/0000-0002-0713-3418

(2)

Maestrante en Psicología Clínica con mención en Psicoterapia por la Universidad Católica de Cuenca.

E-mail: chrisgustavo@hotmail.com Código ORCID:

https://orcid.org/0000-0002-0226-5104

6 Mastrante Psicologí Clinica Maestrante en Psicologia Clínica con mención en Psicoterapia por la Universida Católica de Cuenca.

E-mail: egalarzav@ucacue.edu.ec

digo ORCID:

https://orcid.org/0000-0003-2826-5230

$\mathrm{PhD}$ in Psychology. Faculty of Clinica Psychology, Catholic University of Cuenca, Psychometrics Laboratory of the Center for Research, Innovation and Technology Transfer. E-mail: pmartinezs@ucacue.edu.e Código ORCID:

https://orcid.org/0000-0002-1441-3821

8 Doctor of Neuropsychology. Faculty of Nursing, Catholic University of Cuence Nursing, Catholic University of Cuenca,
Psychometrics Laboratory of the Center for Psychometrics Laboratory of the Center fo E-mail: andres.ramirez@ucacue.edu.ec Código ORCID:

https://orcid.org/0000-0002-6996-0443

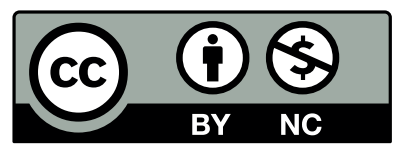

\begin{abstract}
The practice of psychological intervention should be based on the best scientific evidence about its efficacy. The objective of this study was to analyze the attitude, validity and scientific domain possessed by the psychotherapy of Eye Movement Desensitization and Reprocessing (EMDR) based on a scientific realism approach proposed by Bunge in his comparison table of scientific attitudes and pseudoscientific. The methodological design included linguistic and cultural adaptation, content validation with the criteria of judges, from the Bunge table; the bibliographic search of articles between 2016-2020, in the Scopus and Web of Science databases, with experimental and quasi-experimental study designs related to EMDR. The findings evidenced 2417 publications, 38 of which meet the criteria indicated, where it is indicated that EMDR reflects the criteria to be considered a science, sustaining its effectiveness.
\end{abstract}

Keywords:

Science, Pseudoscience, EMDR, Bunge's Table, Validity.

JEL Classification: Z00, Z19.

CITACIÓN: Cedillo, M.L., Viñanzaca, J.P., Espinosa, M.J., Gómez, R.E. \& Mena, C.G. (2021). Análisis del Dominio Científico de la Técnica Psicoterapéutica Eye Movement Desensitization and Reprocessing Desde la Propuesta de Valoración de Mario Bunge. Investigatio, 16, 22-41. doi:10.31095/investigatio.2021.16.3

ENLACE DOI:

http://dx.doi.org/10.31095/investigatio.2021.16.3 


\section{Introducción}

La ciencia atraviesa una etapa compleja al estar siendo cuestionada por las creencias populares, las cuales ante gran parte de la población parecen ser más fuertes que los hechos. Aunque es la objetividad de los hechos la que hace pervivir las teorías. Es común el confundir los pensamientos o creencias en lo "evidente" u obvio con verdades absolutas, y dejar a un lado a los hechos apoyados por "pruebas" o evidencia, la cual indica si una creencia o proposición es verdadera, pues generalmente se encuentra debidamente recogida y analizada (González-Méijome, 2017). Una forma confiable de confirmarlo es a través del método científico (Bunge, 2013). De la confusión entre creencias y hechos, nace un concepto destacado: la pseudociencia (González-Méijome, 2017), que en palabras de Solbes (2019) son conocimientos o creencias que se presentan como científicas, sin serlo, para adquirir una mayor legitimidad.

La Psicología como ciencia nace en el siglo XIX, cuando Wilhem Wundt decide fundar el primer laboratorio de Psicología experimental en el mundo (Greenwood, 2020) hecho a partir del cual muchos otros investigadores emprenden estudios formales y conforman así las diversas escuelas psicológicas. Lightner Witmer en 1896 fundó la Psicología clínica, y a causa del aumento de las demandas de atención durante las dos primeras guerras mundiales, permitió su fortalecimiento y promovió una nueva identidad, que propició la transición del diagnóstico a la intervención psicoterapéutica (Vargas, 2015). Desde este último concepto, podemos hablar de las diversas psicoterapias que se promulgan en nuestros días, encontrando una cantidad de entre 300 a 400 modelos terapéuticos (Balarezo, 2010) los mismos que son objeto de estudio y de los cuales se pone en consideración su cientificidad o no.

Para la investigación en Psicología es importante contar con instrumentos confiables pero también validados, ya sea de constructo, criterio y contenido, esta última es la utilizada en este trabajo para presentar una propuesta de la tabla de Bunge (2010), adaptada desde una perspectiva lingüística y cultural en el contexto ecuatoriano y validada por criterio de expertos; la misma que fue utilizada en el presente artículo para tomar una actitud crítica sobre ciencia y pseudociencia de la técnica EMDR.

La tabla de Bunge se origina del esfuerzo encaminado a la distinción entre la ciencia y la pseudociencia, pues indicó la necesidad de que cualquier proposición científica pueda ser contrastable o estar sujeta a ser falsificada (Bunge, 2012). De tal manera, Bunge propone una taxonomía mediante la cual, desde un enfoque altamente objetivo, se puede verificar las actitudes y actividades típicas que se asocian al dominio científico o pseudocientífico, y tiene trascendencia en varios campos de la ciencia, pues su uso se reporta en el área de la administración (Pineda-Henao \& Tello-Castrillón, 2018), ciencias de la información (Silva et al., 2016), pedagogía (Sáez, 2017), comunicación social (López, 2018), entre otras áreas. De tal manera que, este instrumento se constituye como un insumo clave para la diferenciación entre las inclinaciones científicas y las 
pseudocientíficas. Sin embargo, no se reportan adaptaciones lingüísticas ni culturales realizadas de dicha tabla en el contexto latinoamericano.

La EMDR, acorde a la APA, manifiestan que se necesitan pruebas científicas más concretas que respalden los mecanismos propuestos dentro de su tratamiento, ya que en la actualidad de sitúa como controversial. De esto se desprende el debate entre quienes la defienden, manifestando que, para trabajar síntomas postraumáticos, es la ideal (Figueroa et al.,2016); aunque no se sabe bien cómo funciona, parece contribuir al reprocesamiento emocional de los recuerdos traumáticos (Lee et al., 2013) y así lo respaldan revisiones como las de Marín, Guillén y Vergara (2016), señalando la eficacia de EMDR frente a otras técnicas eclécticas y unas mejoras más rápidas cuando se compara con tratamientos farmacológicos o con terapia cognitivoconductual. Por otro lado, a diferencia de otras técnicas, la EMDR no parte de un conocimiento teórico sino de una experiencia personal de su creadora, lo que ha generado muchas controversias sobre la cientificidad de la misma (Marín et al., 2016). A esto se suma, lo expuesto por Caballo y Salazar (2019), en donde se manifiesta que la EMDR, al igual que las pseudociencias son más propensas a ser impulsadas por objetivos ideológicos, culturales o comerciales, pagándose grandes sumas de dinero para acceder a la capacitación exigida por la autora para practicar su técnica.

\section{$E M D R$}

La EMD por sus siglas en inglés (Eyes
Movement Desensitization) nace de una experiencia personal de su creadora, la doctora Francine Shapiro, en 1987, a partir de una observación casual que observó en sus pacientes que ante pensamientos perturbadores sus ojos se movían rápidamente, provocando la disminución de la carga angustiosa que se asociaba a esos pensamientos (Luber y Shapiro, 2009). Con este descubrimiento, Shapiro emprende estudios con la finalidad de someter a prueba la técnica en memoria traumática, proponiendo que la estimulación bilateral a través de movimientos horizontales rápidos es su componente principal y a través del cual la memoria traumática era desensibilizada.

Esta idea fue mayormente estudiada y se demostró posteriormente que cualquier estimulación bilateral (visual, táctil o auditiva) podía producir la desensibilización de estos recuerdos (Cocco y Sharpe, 1993). A este hecho, se sumó la introducción de la reprocesamiento del recuerdo traumático, conduciendo así en 1991 a un cambio del nombre de la técnica, pasando a llamarse Eyes Movement Desensitization and Reprocessing (EMDR). A partir de este momento, y respaldados por el Instituto de EMDR creado en 1990, se construye un extenso marco explicativo de la técnica denominado modelo del procesamiento adaptativo de la información (Shapiro, 2001).

Se trata de una técnica estructurada de ocho fases: historia del paciente, preparación del paciente, evaluación de los aspectos primarios del recuerdo, desensibilización del recuerdo, instalación de la cognición positiva y reprocesamiento, 
examen corporal, reevaluación y cierre (Shapiro, 1995); cabe recalcar que durante todo el proceso de tratamiento se va reforzando con tareas en casa desde el inicio de la primera sesión (Leeds, 2013).

Una de las partes importantes de este trabajo, es la de exhibir las ventajas que representa el uso de criterios bajo un enfoque de realismo científico, para llevar a cabo un análisis de los ítems de este instrumento y contrarrestarlos con la revisión de la literatura del EMDR. También permitirá dar a conocer la importancia que tiene la identificación de terapias que se encuentren validadas en postulados científicos, distinguiendo de aspectos pseudocientíficos, tal como se establece en párrafos anteriores y acorde a lo manifestado por autores tales como Bunge (2010), así como Caballo y Salazar (2019).

Para ello, Bunge (2010) en su obra denominada "Las pseudociencias ¡vaya timo!" propone una tabla de comparación de las actitudes y actividades de los científicos y pseudocientíficos, en la cual distingue acciones relacionadas a la ciencia tales como apreciar la unidad de la ciencia, utilizar la estadística y la matemática, apoyarse en la lógica, actualizar su conocimiento, aplicar procedimientos objetivos de control, entre otros. Por otra parte, también se encuentran actitudes pseudocientíficas tales como recurrir de manera sistemática al autor, suprime y tergiversa datos no favorables, sus artículos pueden ser entendidos por todos sin necesidad de contar con un lenguaje técnico y pueden adquirir fama muy rápidamente.

\section{Adaptación Lingüistica y Cultural}

Por lo general, los instrumentos desarrollados son específicos para cada una de las culturas y no existen estudios exhaustivos de su aplicabilidad transcultural. Esto resulta relevante cuando es necesario disponer de estrategias de evaluación fiables a la hora de comparar datos de manera inter e intracultural. Para Congost (2010), la adaptación es el proceso de conformar un contenido a la visión particular de cada lengua; intenta crear una equivalencia entre el instrumento original y el instrumento meta con respecto al contenido que es lo que se busca en este estudio.

\section{Validez de Contenido}

Una definición de validez de contenido que supuso la introducción del término en la literatura sobre pruebas educativas y psicológicas (Pedrosa et al., 2013) afirman que si se pretenden validar ítems estadísticamente, se tendría que poder aceptar que el criterio de trabajo es adecuado, para ello, los ítems tendrían que evocar aquello que dicen estar midiendo, una vez establecido este sustento teórico, es cuando surgen los dos criterios fundamentales para estudiar la validez de contenido: relevancia y la coherencia.

El referente metodológico para el proceso de validez se tomó de la propuesta de Escobar y Cuervo (2008) en una plantilla de cuatro categorías: suficiencia, claridad, coherencia y relevancia, donde se ponen en juego una serie de opiniones argumentadas por parte de los expertos y, aunque no se 
encuentren acuerdos unánimes, se pueden identificar las debilidades y fortalezas del instrumento, lo que facilita un análisis profundo respecto a qué modificar, integrar o eliminar del instrumento (Galicia, Balderrama, Navarro, 2017). A pesar de que no se parte de un instrumento psicométrico como tal, sino de un conjunto de criterios, se modela un procedimiento similar al utilizado en la validación de pruebas estandarizadas para la adaptación.

Es por ello, que en este artículo, se presenta la conceptualización de ciencia, pseudociencia, EMDR, validación de contenido y adaptación lingüística, como sustento teórico en la que se asienta la metodología usada. Después, se establece el diseño y las fases con la que se trabajó en la metodología de este estudio. Posteriormente, se establece los resultados del análisis y búsqueda bibliográfica y finalmente, se discuten sus hallazgos y se emiten conclusiones.

\section{Metodología}

Se trata de una revisión sistemática cualitativa, de tipo observacional descriptivo, en donde se resume los hallazgos de ensayos clínicos controlados cuidadosamente seleccionados, lo que propone un alto nivel de evidencia científica de esta intervención acorde a la tabla de Bunge (2010), que se encuentra en su libro "Las pseudociencias ¡vaya timo!" en idioma español. En la revisión de la literatura no se encontraron características psicométricas de esta versión, al no ser una escala, test o inventario que haya pasado por procesos estadísticos para su validación ya que se trata de una tabla comparativa de actitudes y actividades de lo científico vs lo pseudocientífico.

El diseño metodológico incluyó tres fases:

Fase 1: Adaptación Lingüistica y Cultural de la Tabla Bunge (2010) y Validación de Contenido por Criterio de Jueces

Se elaboró una lista de posibles expertos relacionados con el constructo a evaluar y una experta en lengua castellana. Para ello se consideró el cumplimiento de los siguientes criterios de inclusión: haber prestado servicios como profesional durante 10 años o más como docente investigador a nivel universitario, tener formación de $\mathrm{PhD}$ o estar cursando un doctorado; para la determinación del experto en lengua se consideró ser graduado de la especialidad de licenciatura en Español, en una universidad ecuatoriana; haber trabajado en actividades docentes o investigativas para el desarrollo de la lengua castellana durante 10 años o más. De los ocho jueces en total dos son mujeres y seis son hombres.

Para la validación de contenido se contó con la colaboración de diversos investigadores, un primer grupo (cuatro) fueron elegidos entre los profesores del Centro de Investigación de la Universidad Católica de Cuenca con formación académica y profesional en Biología Molecular, Bioquímica, Física y Humanidades; un segundo grupo (tres) con experiencia laboral como psicoterapeutas y docentes de la carrera de Psicología, dos de la misma universidad, una con formación académica y profesional como 
psicoanalista y el otro con formación en psicoterapia del niño y la familia y orientación sistémica, el séptimo juez con formación en Psicología experimental y Neurociencias, docente de la Universidad de Cuenca. Para la adaptación lingüística y cultural se eligió a una docente de la Universidad Católica de Cuenca de la unidad académica de Educación con formación académica en Lengua española y una experiencia de 25 años; dando un total de ocho jueces, según los requerimientos de Escobar y Cuervo (2008).

Se solicitó a cada experto que, de manera individual, realizara una primera revisión de cada ítem, en donde la lingüista realizó la respectiva búsqueda y propuesta de términos que se adecuen al ámbito nacional. Para el análisis de validez de contenido, posterior a su consentimiento informado, se entregó a cada experto una planilla, la misma que contempló una sección dedicada a recoger sus datos personales y profesionales, se describió el objetivo de cada evaluación, así como el cuestionario, su estructura y contó con una hoja de respuestas conforme a los cuatro atributos propuestos por Escobar y Cuervo (2008): claridad, coherencia, relevancia y suficiencia, la escala de medición del atributo se dio con base a una escala tipo likert con cuatro opciones de respuesta 1. Muy bajo, 2. Bajo, 3. Moderado y 4. Alto.

Con la información obtenida, se procedió a la búsqueda de consenso de las sugerencias, y posteriormente al procesamiento estadístico del criterio de jueces a través del coeficiente de la $\mathrm{V}$ de Aiken (1985), quien propuso que el valor mínimo para aceptar un ítem como valido debería de ser de 0.69; Merino-Soto y Livia-Segovia (2009) indican que el cálculo de los intervalos de confianza para este coeficiente permite probar si la magnitud obtenida del coeficiente es superior a una que es establecida como mínimamente aceptable (0.70) para concluir sobre la validez de contenido de los ítems.

Para esta investigación se consideró adecuada si la $\mathrm{V}$ de Aiken era igual o mayor a 0.7 en cada uno de los atributos evaluados: suficiencia, claridad, coherencia y relevancia. Se procede a la nueva propuesta de la tabla propuesta por Bunge (2010).

\section{Fase 2: Búsqueda Bibliográfica}

Se realizó una revisión de los artículos sobre la técnica EMDR publicados desde enero de 2015 hasta febrero de 2020. Se utilizaron las bases de datos de Scopus y Web of Science, introduciendo como palabras clave EMDR y Eye Movement Desensitization and Reprocessing; se obtuvieron 2417 artículos de los cuales se seleccionaron como muestra 38 considerando los siguientes criterios de inclusión: años 2016-2020, diseños de estudio experimentales y cuasiexperimentales, tener los indicadores de la tabla Bunge sobre ciencia y pseudociencia. Estos criterios se han considerado teniendo en cuenta información reciente y actualizada de los últimos cinco años, así como estudios empíricos que reflejen la realidad de la EMDR.

\section{Fase 3: Revisión Sistemática (RS)}

Se realizaron revisiones cualitativas presentando la evidencia en forma 
"descriptiva" y sin análisis estadístico, las mismas que permitieron recopilar y proporcionar estudios sobre el EMDR orientados a responder la pregunta de investigación:

¿La EMDR, es una psicoterapia con bases científicas o pseudocientíficas?

Se utilizaron los pasos para una revisión sistemática de Davies y Crombie (2009), tales como: 1. Definir la pregunta de investigación claramente; 2 . Especificación de los criterios de inclusión y exclusión de los estudios; 3. Formulación del plan de búsqueda de la literatura; 4. Registro de datos y evaluación de la calidad de los estudios seleccionados; y 5. Interpretación y presentación de los resultados.

\section{Resultados}

\section{Adaptación de la Tabla de Bunge}

Posterior a la adaptación lingüística, y

Tabla 1.

Adaptación de la tabla de Bunge

\begin{tabular}{|c|c|c|c|c|}
\hline $\mathbf{N}^{\circ}$ & Tabla original & Tabla adaptada & Coherencia & Relevancia \\
\hline 1 & $\begin{array}{l}\text { Admite su propia ignorancia y, por } \\
\text { ende, la necesidad de mayor } \\
\text { investigación. }\end{array}$ & $\begin{array}{l}\text { Admite su propia ignorancia } \mathrm{y} \text {, por } \\
\text { ende, la necesidad de mayor } \\
\text { investigación. }\end{array}$ & 0.92 & 0.90 \\
\hline 2 & $\begin{array}{l}\text { Considera que su propio campo es } \\
\text { difícil y está lleno de lagunas. }\end{array}$ & $\begin{array}{l}\text { Considera que su propio campo es } \\
\text { difícil y está lleno de lagunas. }\end{array}$ & 0.71 & $0.66 * *$ \\
\hline 3 & $\begin{array}{l}\text { Avanza mediante el planteamiento y } \\
\text { la resolución de nuevos problemas. }\end{array}$ & $\begin{array}{l}\text { Avanza mediante el planteamiento y la } \\
\text { resolución de nuevos problemas. }\end{array}$ & 1.00 & 1.00 \\
\hline 4 & $\begin{array}{l}\text { Recibe con agrado nuevas hipótesis } \\
\text { y métodos. }\end{array}$ & $\begin{array}{l}\text { Recibe con agrado nuevas hipótesis y } \\
\text { métodos. }\end{array}$ & 0.90 & 1.00 \\
\hline 5 & Propone y ensaya nuevas hipótesis. & $\begin{array}{l}\text { Tiene una metodología que satisface la } \\
\text { pregunta de investigación en forma de } \\
\text { hipótesis. }\end{array}$ & 1.00 & 1.00 \\
\hline 6 & Intenta descubrir o aplicar leyes. & Intenta descubrir o aplicar leyes. & 0.95 & 0.90 \\
\hline 7 & Aprecia la unidad de la ciencia. & Eliminada* & 0.47 & 0.66 \\
\hline 8 & Se apoya en la lógica. & Se apoya en la lógica. & 0.81 & 0.81 \\
\hline 9 & Utiliza la matemática. & Utiliza la matemática. & 0.81 & 0.85 \\
\hline 10 & $\begin{array}{l}\text { Recoge o utiliza datos, } \\
\text { especialmente cuantitativos. }\end{array}$ & $\begin{array}{l}\text { Recoge o utiliza datos, cuantitativos y } \\
\text { cualitativos. }\end{array}$ & 0.95 & 0.90 \\
\hline 11 & Busca contraejemplos. & $\begin{array}{l}\text { Busca ejemplos de excepciones a las } \\
\text { reglas propuestas. }\end{array}$ & 0.90 & 0.85 \\
\hline 12 & $\begin{array}{l}\text { Inventa o aplica procedimientos } \\
\text { objetivos de control. }\end{array}$ & $\begin{array}{l}\text { Inventa o aplica procedimientos } \\
\text { objetivos de control. }\end{array}$ & 0.85 & 0.85 \\
\hline 13 & $\begin{array}{l}\text { Resuelve las disputas por medio del } \\
\text { experimento o el cálculo. }\end{array}$ & $\begin{array}{l}\text { Resuelve las disputas por medio del } \\
\text { experimento o el cálculo y aplica } \\
\text { estadística inferencial. }\end{array}$ & 0.81 & 0.81 \\
\hline 14 & $\begin{array}{l}\text { Recurre de manera sistemática a la } \\
\text { autoridad. }\end{array}$ & $\begin{array}{l}\text { Recurre de manera sistemática a la } \\
\text { autoridad. }\end{array}$ & 0.76 & 0.76 \\
\hline 15 & $\begin{array}{l}\text { Suprime o tergiversa los datos no } \\
\text { favorables. }\end{array}$ & $\begin{array}{l}\text { Suprime o tergiversa los datos no } \\
\text { favorables. }\end{array}$ & 1.00 & 0.95 \\
\hline 16 & Actualiza su información. & Actualiza su información. & 0.95 & 1.00 \\
\hline 17 & Busca comentarios críticos de otros. & Busca comentarios críticos de otros. & 0.95 & 0.85 \\
\hline 18 & $\begin{array}{l}\text { Escribe artículos que pueden ser } \\
\text { entendidos por cualquier persona. }\end{array}$ & $\begin{array}{l}\text { Escribe artículos que pueden ser } \\
\text { entendidos por cualquier persona. }\end{array}$ & 0.85 & 0.81 \\
\hline 19 & $\begin{array}{l}\text { Es probable que adquiera fama } \\
\text { instantáneamente. }\end{array}$ & $\begin{array}{l}\text { Es probable que adquiera fama } \\
\text { instantáneamente. }\end{array}$ & 1.00 & 1.00 \\
\hline
\end{tabular}

Nota: *Debido a su baja confiabilidad y validez, se eliminó. **Se mantuvo por el valor del subcriterio "coherencia", excepcionalmente. 
la validez de contenido a través de expertos, se obtuvieron promedios por parte de cada ítem utilizando el coeficiente $\mathrm{V}$ de Aiken, en dónde se obtuvieron 18 ítems con una validez, superior a 0.7 , se elimina el ítem siete (Aprecia la unidad de la ciencia), por presentar una validez menor a 0.6 , en coherencia y relevancia. En el atributo suficiencia se obtiene 0.81 para ciencia y 0.90 para pseudociencia, por lo que no fue necesario agregar nuevos ítems.

\section{Selección de Estudios}

La Tabla 2 evidencia el número de registros identificados, seleccionados $\mathrm{y}$ analizados, tras búsqueda en Scopus y Web of Science. La búsqueda bibliográfica a través de las palabras claves, permitió identificar 2417 registros en las bases de datos. Tras la aplicación del criterio de últimos cinco años, se redujo esta cantidad $(n=1094)$. Estos fueron analizados a partir su título $\mathrm{y}$ resumen, en dónde se pudo identificar el diseño del estudio, escogiendo aquellos experimentales y cuasiexperimentales $(n=211)$, los cuales se analizaron a texto completo, escogiendo aquellos que presenten los indicadores de la Tabla de
Bunge $(n=77)$ y once fueron excluidos por no presentar un alto impacto de publicación (número de citaciones), quedando un total de 51, de las cuales se escogieron 38 en la que de manera explícita se relaciona el ítem respectivo.

\section{Revisión Sistemática}

Las fuentes consultadas acorde a cada indicador, se encuentran en la Tabla 3, en donde se puede observar que los 14 ítems que conforman el dominio científico, fueron apoyados por estudios $(n=37)$, mientras el dominio pseudocientífico, se conformó por cuatro indicadores, de los cuales, no se encontró la evidencia.

\section{Discusión}

Al agrupar los resultados de la dimensión ciencia, los ítems admite su propia ignorancia $\mathrm{y}$, por ende, la necesidad de mayor investigación, y avanza mediante el planteamiento y la resolución de nuevos problemas, los resultados obtenidos indican que estos criterios se sustentan al aplicar EMDR a ciertas patologías. Así, permite ser puesta a prueba su eficacia en otros trastornos (De Bont et al., 2016; ter Heide et al.,

Tabla 2.

Criterios para la búsqueda bibliográfica de los indicadores de tabla de Bunge

\begin{tabular}{|c|c|c|c|c|}
\hline Criterios de inclusión & & Scopus & $\begin{array}{c}\text { Web } \\
\text { of Science }\end{array}$ & Total \\
\hline Palabras claves & EMDR, therapy & 1049 & 1368 & 2417 \\
\hline Últimos cinco años & 2016-2020 & 588 & 506 & 1094 \\
\hline Diseño de estudio & $\begin{array}{l}\text { experimental/ } \\
\text { cuasi experimental }\end{array}$ & 114 & 97 & 211 \\
\hline Tabla de Bunge & Indicadores & 31 & 46 & 77 \\
\hline Seleccionadas & Usadas & 15 & 23 & 38 \\
\hline
\end{tabular}


Tabla 3

Evidencia bibliográfica con base a los indicadores de la Tabla de Bunge

\begin{tabular}{lll}
\hline Dominio & \multicolumn{1}{c}{ Indicadores } & \multicolumn{1}{c}{ Evidencia** } \\
\hline Científico & Admite su propia ignorancia y, & "Se necesita más investigación, preferiblemente con \\
por ende, la necesidad de mayor & medidas continuas para evaluar las HVA, para examinar si \\
investigación. & nuestra falta imprevista de efecto del tratado de TEPT en \\
& las alucinaciones verbales puede ser replicada" (De Bont et \\
& al.,2016). & \\
& "En este estudio, no se encontraron diferencias de \\
& seguridad o eficacia entre la terapia EMDR y la \\
& estabilización como es habitual." (Ter Heide, Mooren et \\
& al., 2016). \\
& "los autores señalan la actual falta de validez en algunas \\
& escalas transculturales de TEPT y aunque este desafío es \\
& bien reconocido" (Tribe et al., 2019).
\end{tabular}

Considera que su propio campo es "Esto no significa que la EMDR esté libre de distorsiones difícil y está lleno de lagunas. de memoria per se. Después de todo, recordar un recuerdo (como se hace en EMDR) es inherentemente un proceso reconstructivo, y las distorsiones en forma de desinformación pueden deslizarse en cualquier momento" (Van Schie., 2016)

"Se calculó el riesgo relativo para el estado de diagnóstico. Se calcularon los intervalos de confianza del $95 \%$ para todos los resultados" (Roberts et al., 2019).

"No se encontró el efecto del estudio original de que la fabricación de EM durante el recuerdo aumenta la susceptibilidad de una persona a endosar información errónea" (Van Schie et al., 2016).

Avanza mediante el "El EMDR parece ser un tratamiento beneficioso para el
planteamiento y la resolución de TEPT en los refugiados sirios, incluso cuando todavía nuevos problemas. están en un campo de refugiados y experimentan altos niveles de trauma continuo" (Acarturk et al., 2016).

"Los meta-análisis de esta revisión proporcionan algunas pruebas de la efectividad de las terapias psicológicas en la prevención del TEPT y la reducción de los síntomas en niños y adolescentes expuestos a un trauma hasta un mes." (Gillies et al., 2016).

"La investigación ha demostrado que hacer EMDR no sólo reduce las clasificaciones auto-reportadas de la vivacidad y/o la emocionalidad de los recuerdos autobiográficos desagradables" (Haerizadeh et al., 2019).

Recibe con agrado nuevas "La terapia EMDR afirma que después del tratamiento de hipótesis y métodos una memoria traumática, el procesamiento de la información es mejorado y se forjan nuevas asociaciones, lo que resulta en un nuevo aprendizaje, la eliminación de la angustia emocional, y el desarrollo de la comprensión cognitiva" (Proudlock et al, 2020).

"Los estudios apoyan que EMDR es efectivo para los síntomas del desorden de estrés post-traumático. También se ha aplicado a otros trastornos porque puede ayudar a resolver y reprocesar los recuerdos de experiencias traumáticas que pueden contribuir" (Balbo et al., 2019).

"En futuros estudios se podrían examinar más a fondo estos resultados añadiendo un grupo de control que permita comparar los efectos de la EMDR con los efectos de un plan de tratamiento alternativo " (Schwarz et al., 2019). 


\begin{tabular}{|c|c|c|}
\hline Dominio & Indicadores & Evidencia** $^{*}$ \\
\hline Científico & $\begin{array}{l}\text { Tiene una metodología } \\
\text { satisface la pregunta } \\
\text { investigación en forma de } \\
\text { hipótesis. }\end{array}$ & $\begin{array}{l}\text { "Este estudio se centra en el potencial de la terapia de } \\
\text { desensibilización y reprocesamiento de los movimien- } \\
\text { tos oculares (EMDR), como una intervención adicio- } \\
\text { nal al tratamiento regular de la adicción" (Markus et } \\
\text { al., 2019). } \\
\text { "Además de los numerosos estudios de control de } \\
\text { casos, ahora también hay disponibles seis ensayos } \\
\text { clínicos controlados aleatorios que demuestran la } \\
\text { eficacia y la seguridad de la EMDR en el tratamiento } \\
\text { de diferentes afecciones de dolor" (Tesarz, Wicking, } \\
\text { Bernardy \& Seidler, (2019). } \\
\text { "Probamos el grado de imposición de WM de varias } \\
\text { condiciones de (no) movimiento ocular utilizadas en } \\
\text { el ensayo clínico". "Resultados: Los ojos en } \\
\text { movimiento son más gravosos que los ojos fijos, } \\
\text { mientras que los ojos fijos no se diferencian de los } \\
\text { ojos desenfocados. Todas las condiciones eran más } \\
\text { exigentes que los ojos cerrados" (Van Veen, Kang, \& } \\
\text { van Schie, 2019). }\end{array}$ \\
\hline
\end{tabular}

Intenta descubrir o aplicar leyes. "Las imágenes guiadas y EMDR podrían reducir el dolor en la artritis reumatoide, pero la reducción del dolor seguía más al EMDR que a las imágenes guiadas" (Ghanbari Nia, Afrasiabifar, \& Behnammoghadam, (2018).

"Este estudio sugiere que EMDR podría ser un tratamiento viable y efectivo para reducir los síntomas depresivos y mejorar la calidad de vida de los pacientes con depresión recurrente" (Ostacoli et al., (2018).

"Tanto la EMDR como la CBT (Terapia Cognitiva Conductual) redujeron los niveles de ansiedad de los pacientes con MI (infarto de miocardio), siendo la primera la más efectiva” (Zeighami, Behnammoghadam, Moradi \& Bashti, (2018).

Se apoya en la lógica.

"La desensibilización y reprocesamiento del movimiento ocular (EMDR) ha contribuido significativamente a la psicoterapia en los últimos 30 años. Los estudios apoyan a EMDR como eficaz para los síntomas del trastorno de estrés postraumático"(Balbo, et al., 2019).

"La mejora clínica en pacientes militares con TEPT después de la EMDR está relacionada con el aumento del metabolismo precario al exponerse al estrés de la RV”. ( Rousseau, Malbos, \& Verger, 2019).

"La eficacia de EMDR en el tratamiento del dolor a través de ensayos clínicos aleatorios Los numerosos estudios de control, hay seis ensayos clínicos controlados aleatorios que demuestran la eficacia y la seguridad de EMDR en el tratamiento de diferentes condiciones de dolor" (Tesarz, Wicking, Bernardy, \& Seidler, 2019). 


\begin{tabular}{|c|c|c|}
\hline Dominio & Indicadores & Evidencia** \\
\hline Científico & Utiliza la matemática. & 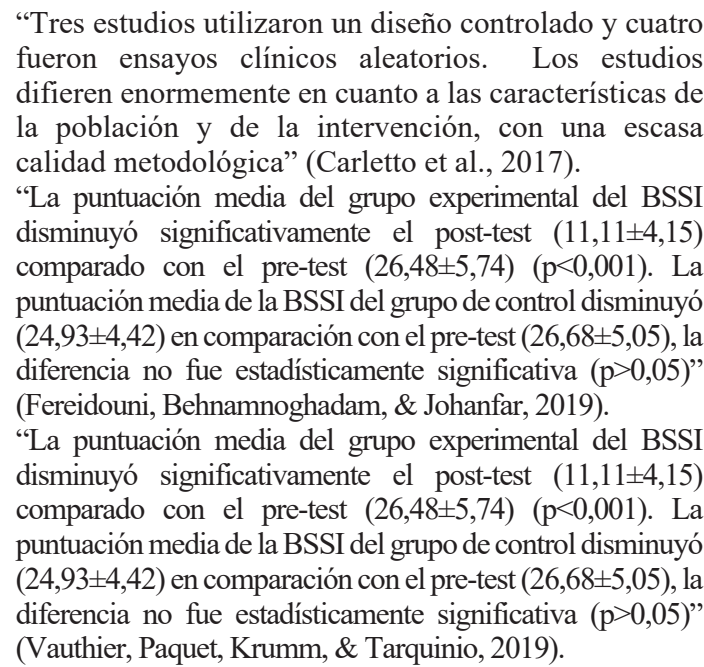 \\
\hline
\end{tabular}

Recoge o utiliza datos, "Los resultados muestran que las clases semánticas
cuantitativos y cualitativos. difieren entre las tres formas de terapia, así como la postura pasiva o activa del paciente durante la terapia" (Brennstuhl, Bassani, \& Tarquino, 2019).

"Se ha demostrado que esa coactivación síncrona da lugar a la formación de una memoria cualitativamente nueva que enlaza las memorias anteriormente independientes" (Chamberlin, 2019).

Busca ejemplos de excepciones a las reglas propuestas.

"Esta revisión analiza tres enfoques terapéuticos para los adolescentes que han sufrido abusos sexuales, entre ellos: la terapia cognitivo-conductual centrada en el trauma, la terapia de juego y la terapia de arte expresivo, el movimiento ocular y la desensibilización, y la terapia de reprocesamiento que proporciona recomendaciones para futuras investigaciones" ( Devli, et al., 2019).

"El creciente interés en los EMDR requiere ensayos controlados aleatorios más sólidos de aplicaciones en trastornos psicopatológicos e investigaciones científicas basadas en la metodología sobre los mecanismos de acción específicos que subyacen a la eficacia clínica de los EMDR en los seres humanos" (Castelnuovo, Fernández, \& Amann, 2019).

Inventa o aplica procedimientos objetivos de control.

"Los participantes en este estudio fueron pacientes consecutivos que fueron diagnosticados con PTSD actual según la Entrevista Clínica Estructurada para los trastornos del Eje I del DSM-IV-Versión Clínica"(Bae, Kim, \& Park, 2016). "Nuestra revisión narrativa proporciona un resumen de los resultados de la investigación para cada familia de tratamiento. Los resultados están organizados por nivel de evidencia para cada familia de tratamiento" (Dorsey et al., 2017).

"Todos los participantes seleccionaron dos recuerdos autobiográficos aversivos y seleccionaron una imagen objetivo para cada recuerdo. Utilizando el contrabalanceo, un recuerdo fue identificado como "memoria relevante" y el otro como "memoria irrelevante". (van Veen, Engelhard, \& Van den Hout, 2016). 


\begin{tabular}{|c|c|c|}
\hline Dominio & Indicadores & Evidencia** \\
\hline \multirow[t]{2}{*}{ Científico } & $\begin{array}{l}\text { Resuelve las disputas por medio } \\
\text { del experimento o el cálculo. } \\
\text { Aplica estadística inferencial. }\end{array}$ & $\begin{array}{l}\text { "En el presente estudio, la administración de base de la } \\
\text { escala arrojó un valor } \alpha \text { de Cronbach de } 0,87 \text {, lo que } \\
\text { indica una buena consistencia interna de todos los } \\
\text { elementos. (Acarturk et al., 2016). } \\
\text { "En un ensayo controlado aleatorio (ECA) a ciegas, } \\
155 \text { pacientes ambulatorios en tratamiento para la } \\
\text { psicosis ( } 61,3 \% \text { de trastorno esquizofrénico, } 29 \% \text { de } \\
\text { trastorno esquizoafectivo) fueron asignados al azar a } \\
\text { ocho sesiones de exposición prolongada (PE; n = 53)" } \\
\text { (de Bont et al., 2016). } \\
\text { "Los estudiantes universitarios con bajo (n31) o alto } \\
\text { (n35) WMC recordaron tres memorias emocionales y } \\
\text { calificaron la vivacidad y la emotividad antes y después } \\
\text { de cada condición (recordar sólo, recordar el EM lento } \\
\text { y recordar el EM rápido)" (Haagen, ter Heide, Mooren, } \\
\text { Knipscheer, \& Kleber, 2016). }\end{array}$ \\
\hline & Actualiza su información. & $\begin{array}{l}\text { "Ir más allá de los límites del diagnóstico, centrándose } \\
\text { en la interacción causal entre procesos traumáticos } \\
\text { específicos y utilizando medidas estandarizadas, son } \\
\text { direcciones útiles para futuras investigaciones sobre la } \\
\text { memoria, las emociones y los traumas emocionales" } \\
\text { (Giotakos, 2020). } \\
\text { "El estudio actual será el primer ECA que examine la } \\
\text { eficacia de la intervención temprana de EMDR en } \\
\text { comparación con el tratamiento habitual para reducir } \\
\text { los síntomas de estrés postraumático en las víctimas de } \\
\text { violación (Covers et al., 2019). } \\
\text { "Este estudio contribuirá a la actualmente limitada base } \\
\text { de pruebas para la EMDR para el TEPT en los entornos } \\
\text { forenses. Es el primer ensayo clínico aleatorio que } \\
\text { evalúa la eficacia, el riesgo de daño y la aceptabilidad } \\
\text { de la EMDR" (Every-Palmer et al., 2019). }\end{array}$ \\
\hline
\end{tabular}

\begin{tabular}{lll}
\cline { 2 - 2 } & $\begin{array}{l}\text { Busca comentarios críticos de } \\
\text { otros. }\end{array}$ & No se evidencia* \\
\hline Pseucientífico & $\begin{array}{l}\text { Recurre de manera sistemática a } \\
\text { la autoridad. }\end{array}$ & No se evidencia* \\
\cline { 2 - 2 } & $\begin{array}{l}\text { Suprime o tergiversa los datos no } \\
\text { favorables. }\end{array}$ & No se evidencia* \\
\hline & $\begin{array}{ll}\text { Escribe artículos que pueden ser } \\
\text { entendidos por cualquier persona. }\end{array}$ & No se evidencia* \\
\hline & $\begin{array}{l}\text { Es probable que adquiera fama } \\
\text { instantáneamente. }\end{array}$ & No se evidencia* \\
\hline
\end{tabular}

Nota: *No se han encontrado artículos que explícitamente denoten el indicador. ** La evidencia responde a una traducción realizada por los autores.

2016; Tribe et al., 2019), como los de ansiedad y de pánico (Acarturk et al., 2016; Gillies et al., 2016; Haerizadeh et al., 2019). Al referir si esta técnica recibe con agrado nuevas hipótesis y métodos, se evidencia en función a los síntomas que presente el paciente (Balbo et al., 2019; Proudlock y Peris, 2020; Schwarz et al., 2019). Inventa o aplica procedimientos objetivos de control, los investigadores de EMDR como: Van Veen et al., (2016) generan experimentos 
con una base teórica fuerte y concluyen que existen resultados positivos en la terapia EMDR, pero hace falta más experimentación para contrastar dichos resultados.

Es importante destacar que se ha comparado la eficiencia de esta técnica con otras terapias como la Terapia cognitiva-conductual y como resultado se encuentra que la EMDR es eficaz pero que no supera a otras técnicas (Dorsey et al., 2017). Al mismo tiempo, Bae et al. (2016), observan que no es eficiente cuando existen comorbilidades psiquiátricas en pacientes adultos. Se apoya en la lógica, al analizar los comportamientos y patrones de los pacientes, aplicando la sistematización de los argumentos para la planificación terapéutica se puede comprobar la utilización de la lógica en sus procedimientos (Balbo et al., 2019; Rousseau et al., 2019; Tesarz et al., 2019). Utiliza la matemática, para evidenciar este criterio se debe considerar que los resultados de la mayoría de las investigaciones han aplicado: anova, correlaciones, estadística inferencial o inductiva, ensayos clínicos aleatorizados, meta análisis (Carletto et al., 2017; Fereidouni et al., 2019; Vauthier et al., 2019).

Recoge o utiliza datos especialmente cuantitativos o cualitativos, la mayoría de los resultados del EMDR arrojan datos cuantitativos los mismos que poseen algún método de investigación, para ello se recurre a análisis de comparaciones numéricas y estadísticas (Brennstuhl et al., 2019; Chamberlin, 2019; van Veen, Kang, \& van Schie, 2019).
Busca ejemplos de excepciones a las reglas propuestas, se necesita profundizar los estudios en especial de los casos clínicos (Devli, et al., 2019; Castelnuovo et al., 2019; Valiente -Gómez et al., 2017). Resuelve las disputas por medio del experimento o el cálculo, las investigaciones realizadas hasta el momento sobre el EMDR han sido variadas intentando responder preguntas sobre la eficacia y para esto es imprescindible el uso de las matemáticas. (De Bont et al., 2016; Acarturk et al., 2016; Haagen, Ter Heide et al., 2016).

Actualiza su información, la EMDR, desde su creación, ha sido objeto de experimentación (Cotter et al., 2017; Covers et al., 2019; Hegarty et al., 2016), a través de sus estudios, han actualizado la información de su eficacia y validez, lo que conlleva la modificación de datos, hipótesis y teorías, incluso con respecto a otros enfoques. Busca comentarios críticos de otros, no se opone a las críticas de otras corrientes o de sus detractores, al contrario, las toma en cuenta para sus estudios de validez y efectividad (Brennstuhl y Bassan, 2018; Jahanfar et al., 2020; Markus et al., 2019).

En cuanto a la EMDR como pseudociencia, cuatro ítems específicos del criterio pseudociencia han sido analizados: Recurre de manera sistemática a la autoridad, en la bibliografía consultada no se muestra que se dirija en particular a ningún autor en específico. Lo que se halló es que muchas investigaciones quieren experimentar o explicar la EMDR desde su corriente psicológica (Pernot-Masson, 2016; 
Brooker, 2018; Haan et al., 2017). Suprime o tergiversa los datos no favorables, Matthijssen y Van den Hout (2016) refieren dos estudios con estudiantes universitarios que no mostraron ningún valor aditivo de la EM (Eye movement) en el procedimiento, pero tampoco ningún efecto contraproducente. Los EM no son efectivos para mejorar la creencia en la posesión de un rasgo de la personalidad o la emocionalidad.

Este estudio muestra una contradicción con los resultados obtenidos en otros experimentos con la EMDR que en su mayoría muestran que es efectiva, además, se realizaron análisis de resultados con una mejoría de síntomas repentinos que no necesariamente tiene que ver con la técnica EMDR (Marsden, et al., 2018; Mevissen, et al., 2017) estos estudios evidencian que EMDR no tergiversa datos no favorables. Escribe artículos que pueden ser entendidos por cualquier persona, a pesar de que la EMDR, se ha situado como una psicoterapia en auge, no se ha encontrado artículos en los que el lenguaje sea superfluo o coloquial, más bien, al contrario, el léxico, así como el nivel de escritura, se encuentra acorde a personas formadas en campos de la salud.

Se evidencia en artículos tales como de Khan et al., (2019), Ghanbari Nia et al., (2018), Van Veen et al., (2019) en los que se utilizan metodologías experimentales y cuasiexperimentales, partiendo de epistemologías objetivas, así como modelos estadísticos inferenciales. Es probable que adquiera fama instantáneamente, bajo estas consideraciones, se observa que la EMDR, ha sido acogida con una gran demanda por parte de los usuarios, especialmente de aquellos que presentan estrés y postrauma, lo cual ha generado fama instantánea (Moghadam et al., 2020; Van den Hout et al., 2011; Van Schie y Leer, 2019) si bien, debería revisarse Bunge (2010) más en profundidad para comprender que no es lo mismo popularidad científica, como es el caso que popularidad a nivel de conocimiento vulgar.

Finalmente, un ítem de la tabla Bunge pese a ser asignado a la dimensión de científico se ha considerado necesario ubicarlo en esta sección, considera que su propio campo es difícil y está lleno de lagunas, para la Asociación Americana de Psicología, aún existen dudas de que la terapia EMDR sea considerada como ciencia, consideran que falta un respaldo científico mayor que avale su eficacia, uno de los impedimentos para denominarla totalmente como una técnica psicoterapéutica científica sería su reciente aparición en el campo de la Psicología y pese a las evidencias sobre su eficacia todavía se encuentra en el punto de mira como objeto de estudio por parte de los expertos (Pernot, 2016; Roberts et al., 2019; van Schie, va Veen et al., 2016).

Tras lo analizado, se observa que se asienta en un dominio científico, sin embargo, en la actualidad es considerada controversial; es así que, vale la pena considerar para que se pueda contrastar la información en futuros estudios y así 
modificar protocolos de la técnica que están siendo criticados.

\section{Conclusiones}

La adaptación lingüística y cultural de la tabla propuesta por Mario Bunge (2010), es necesaria, como instrumento que permita juzgar con parámetros subyacentes en la realidad en que se encuentra siendo analizada.

La validación de cada uno de los ítems de la tabla propuesta por Bunge, a través de expertos con alta formación profesional, permitió identificar desde una perspectiva científica, cuáles son los indicadores con mayor fuerza para diferenciar entre ciencia y pseudociencia en el contexto ecuatoriano

La búsqueda bibliográfica, permitió identificar múltiples estudios experimentales y cuasiexperimentales que respaldan, los criterios de cientificidad de la EMDR, lo cual evidencia cumple con la propuesta de dominios de cientificidad asentados por Bunge.

\section{Conflicto de intereses}

Este artículo no presenta conflicto de intereses entre los autores firmantes.

\section{Agradecimientos}

A la Unidad de Posgrado, al programa de Maestría en Psicología Clínica con Mención en Psicoterapia y al Laboratorio de Psicometría del Centro de Investigación, Innovación y Transferencia de TecnologíaCIITT de la Universidad Católica de Cuenca.

\section{Referencias}

Acarturk, C., Konuk, E., Cetinkaya, M., Senay, I., Sijbrandij, M., Gulen, B., \& Cuijpers, P. (2016). The efficacy of eye movement desensitization and reprocessing for post-traumatic stress disorder and depression among Syrian refugees: Results of a randomized controlled trial. Psychological medicine, 46(12), 2583-2593. DOI: $10.1017 / \mathrm{S} 0033291716001070$

Aiken. L. (1985). Three coefficients for analyzing the reliability and validity of ratings. Educational and Psychological Measurement. 45(1), 131-142 https://doi. org/10.1177/0013164485451012

Bae, H., Kim, D., \& Park, Y. C. (2016). Dissociation predicts treatment response in eye-movement desensitization and reprocessing for posttraumatic stress disorder. Journal of Trauma \& Dissociation, 17(1), 112-130. DOI: 10.1080/15299732.2015.1037039.

Balarezo, L. (2010). Modelo de Psicoterapia Focalizado en la Personalidad. Quito: Centro de Publicaciones PUCE.

Balbo, M., Cavallo, F., \& Fernández, I. (2019). Integrating EMDR in psychotherapy. Journal of Psychotherapy Integration, 29(1), 23. DOI: 10.1037/int0000136

Brennstuhl, M.-J., Bassan, F., \& Tarquinio, C. (2018). Analyse et comparaison de discours de patients douloureux chroniques durant une thérapie de soutien par opposition à l'utilisation de la thérapie Eye Movement Desensitization and Reprocessing. (EMDR). Pratiques Psychologiques, 25(4), 441-451. https://doi.org/10.1016/ j.prps.2018.06.003

Bunge, M. (2010). Las pseudociencias ¡vaya timo!. Navarra: Laetoli.

Bunge, M. (2012). Filosofía para médicos. Barcelona: Gedisa. 
Bunge, M. (2013). La ciencia: su método y su filosofía. Pamplona: Laetoli.

Caballo, V. E., \& Salazar, I. C. (2019). Ingenuos: El engaño de las terapias alternativas. Siglo XXI de España Editores.

Carletto, S., Ostacoli, L., Colombi, N., Luca, C., Francesco, O., Fernandez de castro, I., \& Hofmann, A. (2017). EMDR for depression: A systematic review of controlled studies. Clinical Neuropsychiatry, 306-312.

Castelnuovo, G., Fernandez, I., \& Amann, B. (27 de Septiembre de 2019). Editorial: Present and Future of EMDR in Clinical Psychology and Psychotherapy. Frontiers in Psychology, 10, 2185. doi:10.3389/fpsyg.2019.02185

Chamberlin, E. (2019). The Predictive Processing Model of EMDR. Frontiers in Psychology, 10, 2267. doi:10.3389/fpsyg.2019.02267.

Cocco, N. \& Sharpe, L. (1993). An auditory variant of eye movement desensitization in a case of childhood post-traumatic stress disorder. Journal of Behavior Therapy and Experimental Psychiatry, 24, 373-377. http://dx.doi.org/10.1016/00057916(93)90062-2

Congost N. (2010). El lenguaje de las ciencias de la salud en el ámbito de los lenguajes profesionales y de especialidad [tesis doctoral]. España: Universidad de A 1 i $\begin{array}{llllllll} & \text { c } & \text { a } & n & t & \text { e }\end{array}$. http://rua.ua.es/dspace/bitstream/10045/1 7562/1/Tesis_congost.pdf

Cotter, P., Meysner, L., \& Lee, C. W. (2017). Participant experiences of Eye Movement Desensitisation and Reprocessing vs. Cognitive Behavioural Therapy for grief: similarities and differences. European Journal of Psychotraumatology, 8 (sup6), 1375838. DOI: $10.1080 / 20008198.2017$. 1375838.
Covers, M., Jongh, A., Huntjens, R., De Roos, C., Van Den Hout, M. , \& Bicanic, I. (2019). Early intervention with eye movement desensitisation and reprocessing (EMDR) therapy to reduce the severity of posttraumatic stress symptoms in recent rape victims: study protocol for a randomised controlled trial. European Journal of Psychotraumatology, 10 (1), 1632021. DOI: $10.1080 / 20008198.2019 .1632021$.

Davis, H., \& Crombie, I. What is a systematic review? (sitio web). Disponible en: http://www.evidence-based-medicine. Co.uk/ebmfiles/WhatisSystreview.pdf (consultado el 4/6/2008).

De Bont, P., Van den Berg, D., Van der Vleugel, B., De Roos, C., De Jongh, A., Van Der Gaag, M., \& Van Minnen. (2016). Prolonged exposure and EMDR for PTSD v. a PTSD waiting-list condition: effects on symptoms of psychosis, depression and social functioning in patients with chronic psychotic disorders. Psychological Medicine, 46(11), 2411-2421. doi:10.1017/S0033291716001094.

Devlin, J., Hill, L., Berry, J., Felde, K., \& Wilson, C. (29 de Abril de 2019). Therapeutic Practices for Sexually Abused Children and Adolescents: Resources for Marriage, Family, and Couples' Counselors. The Family Journal, 27(4), 359-365. doi:10.1177/1066480719844017.

Dorsey, S., McLaughlin, K. A., Kerns, S. E., Harrison, J. P., Lambert, H. K., Briggs, E. C. \& Amaya-Jackson, L. (2017). Evidence base update for psychosocial treatments for children and adolescents exposed to traumatic events. Journal of Clinical Child \& Adolescent Psychology, 46(3), 303-330. DOI: 10.1080/15374416. 2016.1220309.

Escobar-Pérez, J. y A. Cuervo-Martínez (2008).Validez de contenido y juicio de expertos: una aproximación a su utilización. Avances en Medición, 6, 27-36. 
Every-Palmer, S., Flewett, T., Dean, S., Hansby, O., Colman, A., Weatherall, M., \& Bell, E. (2019). Eye movement desensitization and reprocessing (EMDR) therapy for posttraumatic stress disorder in adults with serious mental illness within forensic and rehabilitation services: a study protocol for a randomized controlled trial. Trials, 20(1). doi:10.1186/s13063-019-3760-2

Fereidouni, Z., Behnammoghadam, M., Jahanfar, A., \& Dehghan, A. (2019). The Effect of Eye Movement Desensitization and Reprocessing (EMDR) on the severity of suicidal thoughts in patients with major depressive disorder: a randomized controlled trial. Neuropsychiatric Disease and Treatment, 15, 2459-2466. doi:10.2147/NDT.S210757.

Figueroa, R. A., Cortés, P. F., Accatino, L., \& Sorensen, R. (2016). Trauma psicológico en la atención primaria: orientaciones de manejo. Revista médica de Chile, 144(5), 643-655. https://dx.doi.org/10.4067/S003 4-98872016000500013

Galicia Alarcón, L. A., Balderrama Trápaga, J. A., \& Edel Navarro, R. (2017). Validez de contenido por juicio de expertos: propuesta de una herramienta virtual. Apertura (Guadalajara, Jal.), 9(2), 42-53. Doi: 10.18381 .

Ghanbari Nia, N., Afrasiabifar, A., \& Behnammoghadam, M. (2018). Comparing the effect of eye movement desensitization and reprocessing (EMDR) with guided imagery on pain severity in patients with rheumatoid arthritis. Journal of Pain Research, 11, 2107-2113. doi:10.2147/ jpr.s158981.

Gillies, D., Maiocchi, L., Bhandari, A. P., Taylor, F., Gray, C., \& O'Brien, L. (2016). Psychological therapies for children and adolescents exposed to trauma. Cochrane database of systematic reviews, (10). doi.org/10.1002/14651858.CD012371.
González-Méijome, J. M. (2017). Science, pseudoscience, evidence-based practice and post truth. Journal of Optometry, 10(4), 203-204. doi:10.1016/j.optom. 2017.08.001.

Greenwood, J. D. (2020). Sobre dos principios fundamentales de la Escuela de Psicología Gestalt de Berlín. Revisión de Psicología General, 1089268019893972.

Giotakos, O. (2020). Neurobiology of emotional trauma. Psychiatriki, 31(2), 162-171. 10.22365/jpsych.2020.312.162

Haagen, J., Ter Heide, F., Mooren, T., Knipscheer, J., \& Kleber, R. (2016). Blurring emotional memories using eye movements : individual differences and speed of eye movements. European Journal of Psychotraumatology, 7 (1), 29476. DOI: 10.3402/ejpt.v7.29476.

Haan, K., Lee, C., Fassbinder, E., Voncken, M. Meewisse, M., Van Es, S. Menninga, S. Kousemaker, M. \& Arntz, A. (2017). Imagery rescripting and eye movement desensitisation and reprocessing for treatment of adults with childhood trauma-related post-traumatic stress disorder: IREM study design. $B M C$ Psychiatry, 17(1). DOI: 10.1186/s12888017-1330-2.

Haerizadeh, M., Sumner, J. A., Birk, J. L., Gonzalez, C., Heyman-Kantor, R., Falzon, L., \& Kronish, I. M. (2019). Interventions for posttraumatic stress disorder symptoms induced by medical events: A systematic review. Journal of Psychosomatic Research, 109908. doi.org/10.1016/ j.jpsychores.2019.109908

Hegarty, K., Tarzia, L ., Hooker, L ., \& Taft, A. (2016) Interventions to support recovery after domestic and sexual violence in primary care. International Reviuw of Psychiatry, 28(5), 519-532. DOI: 10.1080/09540261.2016.1210103.

Hernández-Nieto, R. A. (2002). Contributions to 
Statistical Analysis. Mérida, Venezuela: Universidad de Los Andes.

Jahanfar, A., Fereidouni, Z., Behnammoghadam, M., Dehghan, A., \& Bashti, S. (2020). Efficacy of Eye Movement Desensitization and Reprocessing on the Quality of Life in Patients with Major Depressive Disorder: A Randomized Clinical Trial. Psychology Research and Behavior Management, 13, 11-17. doi:10.2147/prbm.s232589.

Khan, A., Khan, S., \& Shah, S. T. (2019). Efficacy of Eye Movement Desensitization \& Reprocessing Versus Cognitive Behavioral Therapy in Post-Traumatic Stress and Depressive Symptoms: Study protocol for a Randomized Controlled Trial. Contemporary Clinical Trials Communications, 16, 100439. doi:10.1016/j.conctc.2019.100439.

Lee, C. W., \& Cuijpers, P. (2013). A meta-analysis of the contribution of eye movements in processing emotional memories. J Behav Ther Exp Psychiatry 44 (2), 231-9

Leeds, A. M. (2013). Guía de protocolos estándar de EMDR para terapeutas, supervisores y consultores. Desclée de Brouwer.

López, B. M. (2018). The use of pseudoscience and experimentation as a persuasive resource in new advertising communication trends. Revista Latina De Comunicación Social, (73), 1428-1443. doi:10.4185/ RLCS-2018-1315-74

Luber, M. y Shapiro, F. (2009). Interview with Francine Shapiro: Historical overview, present issues, and future directions of EMDR. Journal of EMDR Practice and Research, 3, 217-231.

Marín, C., Guillén, A. I., \& Vergara, S. (2016). Nacimiento, desarrollo y evolución de la desensibilización y el reprocesamiento por medio de movimientos oculares (EMDR). Clínica y Salud, 27(3),
101-114. https://dx.doi.org/10.1016/j.cly sa.2016.09.001

Markus, W., Hornsveld, H. K., Burk, W. J., de Weert-van Oene, G. H., Becker, E. S., \& DeJong, C. A. J. (2019). Addictionfocused Eye Movement Desensitization and Reprocessing Therapy as an Adjunct to Regular Outpatient Treatment for Alcohol Use Disorder: Results from a Randomized Clinical Trial. Alcoholism. Clinical and Experimental Research, 44(1), 272-283. Doi:10.1111/acer.14249.

Marsden, Z. Teahan, A., Lovell, K., Blore, D., \& Delgadillo, J. (2018). Patients' experiences of cognitive behavioural therapy and eye movement desensitisation and reprocessing as treatments for obsessive-compulsive disorder. Couselling and Psychoterapy Research, 18(3), 251-261 DOI: 10.1002/capr.12159.

Matthijssen, S., \&Van den Hout, M. (2016) The use of EMDR in positive verbal material: results from a patient study. European Journal of Psychotraumatology, 7(1), 30119, DOI: 10.3402/ejpt.v7.30119

Merino, C., \& Livia, J. (2009). Intervalos de confianza asimétricos para el índice la validez de contenido: Un programa Visual Basic para la V de Aiken. Anales de Psicología, 25(1), 169-171. Recuperado de: http://www.redalyc.org/articulo.oa?id $=16711594019$

Mevissen, L., Didden, R., Korzilius, H., Hubert., \& De Jongh, A. (2017).Eye movement desensitisation and reprocessing therapy for posttraumatic stress disorder in a child and an adolescent with mild to borderline intellectual disability: A multiple baseline across subjects study. Journal of Applied Research in Intellectual Disabilities, 30, 34-41. DOI: 10.1111/jar.12335

Moghadam, S. A., Kazemi, R., Taklavi, S., \& Naeim, M. (2020). Comparing the 
effectiveness of eye movement desensitization reprocessing and cognitive behavioral therapy in reducing post-traumatic stress disorder. Health Psychology Report, 8(1), 31-37.

Ostacoli, L., Carletto, S., Cavallo, M., BaldomirGago, P., Di Lorenzo, G., Fernandez, I., \& Oliva, F. (2018). Comparison of eye movement desensitization reprocessing and cognitive behavioral therapy as adjunctive treatments for recurrent depression: The European Depression EMDR Network (EDEN) randomized controlled trial. Frontiers in psychology, 9, 74. doi.org/10.3389/fpsyg.2018.00074

Pedrosa, Ignacio, Suárez-Álvarez, Javier, \& GarcíaCueto, Eduardo. (2013). Evidencias sobre la validez de contenido: avances teóricos y métodos para su estimación. Acción Psicológica, 10(2), 3-18. https://dx.doi.org/10.5944/ap.10.2.11820

Pernot-Masson, A. (2016). Traumatismes et perversions des relations parents-enfants. La Psychiatrie de L'enfant, 59(1), 97. DOI:10.3917/psye.591.0097.

Pineda-Henao, E., \& Tello-Castrillón, C. (2018). ¿Ciencia, técnica y arte?: Análisis crítico sobre algunas posturas del problema del estatus epistemológico de la administración. [science, technique and art? critical analysis of some streams of the epistemological status of management]. Revista Logos, Ciencia \& Tecnología, 10(4), 112-130. https://search-proquest-com.vpn.ucacue.e du.ec/docview $/ 2166520015$ ? accountid $=6$ 1870

Proudlock, S., \& Peris, J. (2020). Using EMDR therapy with patients in an acute mental health crisis. BMC Psychiatry, 20(1). doi:10.1186/s12888-019-2426-7

Roberts, N. P., Kitchiner, N. J., Kenardy, J., Lewis, C. E., \& Bisson, J. I. (2019). Early psychological intervention following recent trauma: A systematic review and meta-analysis. European Journal of Psychotraumatology, 10(1), 1695486. doi.org/10.1080/20008198.2019.1695486

Rousseau, M. V. (2019). Increase of precuneus metabolism correlates with reduction of PTSD symptoms after EMDR therapy in military veterans: an 18 F-FDG PET study during virtual reality exposure to war. European Journal of Nuclear Medicine and Molecular Imaging, 46(9), 1817-1821. doi:10.1007/s00259-019-04360-1

Sáez Alonso, R. (2017). La prioridad del método en la investigación pedagógica/The priority of method in pedagogical research. Revista española de pedagogía, 239-254.

Shapiro, F. (2001). EMDR: Basic principles, protocols and procedures. Nueva York: Guilford Press.

Shapiro, F. (1995). Eye movement desensitization and reprocessing: Basic principles, protocols, and procedures. Nueva York: Guilford Press.

Schwarz, J. E., Baber, D., Barter, A., \& Dorfman, K. (2020). A mixed methods evaluation of EMDR for treating female survivors of sexual and domestic violence. Counseling Outcome Research and Evaluation, 11(1), 4-18. doi.org/10.1080/21501378.2018.1561146

Silva, L. M., Vianna, W. B., \& Kern, V. M. (2016). O sistemismo de bunge como base teórico-metodológica para pesquisa em ciência da informação. Em Questão, 22(2), 140-164. Retrieved from https://search-proquest-com.vpn.ucacue.e du.ec/docview $/ 1813877804$ ?accountid $=6$ 1870

Solbes, J. (2019). Cuestiones socio-científicas y pensamiento crítico: Una propuesta para cuestionar las pseudociencias. Tecné, Episteme y Didaxis: TED, (46), 81-99. http://www.scielo.org.co/scielo.php?pid= 
S012138142019000200081\&script $=$ sci abstract\&tlng=pt

Ter Heide, F. J. J., Mooren, T. M., Van de Schoot, R., De Jongh, A., \& Kleber, R. J. (2016). Eye movement desensitisation and reprocessing therapy v. stabilisation as usual for refugees: Randomised controlled trial. The British Journal of Psychiatry, 209(4), 311-318. DOI: https://doi.org/10.1192/bjp.bp.115.167775

Tesarz, J., Wicking, M., Bernardy, K., \& Seidler, G. H. (2019).EMDR Therapy's Efficacy in the Treatment of Pain.Journal ofEMDR Practice and Research, 13(4), 337-344

Tribe, R. H., Sendt, K. V., \& Tracy, D. K. (2019). A systematic review of psychosocial interventions for adult refugees and asylum seekers. Journal of Mental Health, 28(6), 662-676. doi.org/10. $1080 / 09638237.2017 .1322182$

Valiente, A., Moreno, A., Treen, D., Cedrón, C., Colom, F., Pérez, V., \& Amann, B. (2017). EMDR beyond PTSD: A Systematic Literature Review. Frontiers in Psychology, 8, 1668. doi:10.3389/ fpsyg.2017.01668

Van den Hout, M. A., Engelhard, I. M., Rijkeboer, M. M., Koekebakker, J., Hornsveld, H., Leer, A., (...) Akse, N. (2011). EMDR: Eye movements superior to beeps in taxing working memory and reducing vividness of recollections. Behaviour Research and Therapy, 49(2), 92-98.

Van Schie, K., \& Leer, A. (2019). Lateral Eye Movements Do Not Increase False-Memory Rates: A Failed Direct-Replication Study. Clinical Psychological Science, 7(5), 1159-1167. $\begin{array}{lllllllllllllll}2 & 1 & 6 & 7 & 7 & 0 & 2 & 6 & 1 & 9 & 8 & 5 & 9 & 3 & 3\end{array}$. doi:10.1177/2167702619859335

Van Schie, K., van Veen, S. C., Engelhard, I. M., Klugkist, I., \& van den Hout, M. A. (2016). Blurring emotional memories using eye movements: Individual differences and speed of eye movements. European Journal of Psychotraumatology, 7(1), 29476. doi.org/10.3402/ejpt.v7.29476

Van Veen, S. C., Kang, S., \& van Schie, K. (2019). On EMDR: Measuring the working memory taxation of various types of eye (non-)movement conditions. Journal of Behavior Therapy and Experimental Psychiatry, 65, 101494. doi:10.1016/j. jbtep.2019.101494

Van Veen, S. C., Engelhard, I. M., \& van den Hout, M. A. (2016). The effects of eye movements on emotional memories: Using an objective measure of cognitive load. European Journal of Psychotraumatology, 7(1), 30122. DOI: 10.3402/ejpt.v7.30122

Vargas, M. G. (2015). Concepto de psicoterapia en psicología clínica. Revista de psicología Universidad de Antioquia, 2(2), 19-32.

Vauthier, M., Paquet, Y., Krumm, C., \& Tarquinio, C. (2019). Towards a new understanding of mathematics through Eyes Movement Desensitization and Reprocessing: News and perspectives. Psychologie Française, 64(3), 295-304. doi:10.1016/j.psfr.2017. 11.001

Zeighami, R., Behnammoghadam, M., Moradi, M., \& Bashti, S. (2018). Comparison of the effectof eyemovement desensitization reprocessing and cognitivebehavioral therapy on anxiety in patients withmyocardial infarction. The European Journal of Psychiatry,32(2), 72-76. 